\title{
Are we good enough in the evaluation of MPI using Rubidium82 with PMT PET/CT? A comparison to SiPM PET/CT
}

\author{
Christel H. Kamani, $M D{ }^{a}$ and John Prior, PhD, $M D^{a, b}$ \\ a Department of Nuclear Medicine and Molecular Imaging, CHUV, Lausanne, Switzerland \\ b Faculty of Biology and Medicine, Lausanne University Hospital, University of Lausanne, \\ Lausanne, Switzerland
}

Received Apr 21, 2020; accepted Apr 21, 2020

doi: 10.1007/s12350-020-02169-2

\section{See related article, pp. 204-212}

The success of nuclear cardiology relies on the use of a combination of highly specific signals provided by radiotracers, as well as the use of improved electronic nuclear instrumentation to specifically trace in-vivo the metabolic reaction of interest. Since the development of the first clinical positron imaging device by Brownell and Aronow in 1953, incredible progresses have been made. Some of these progresses involved the fusion of PET and CT in an hybrid device, the development of full-ring PET as well as the discovery of fast ceriumdoped lutetium oxyorthosilicate (LSO) with interesting physical properties allowing the clinical implementation of time-of-flight (TOF) technology. ${ }^{1}$ This constant query for modern devices with sophisticated reconstruction algorithms has clinical and therapeutic implications, since PET/CT provides semi-quantitative, as well as quantitative parameters of the myocardial blood flow (MBF). These parameters are used as "gatekeeper" for coronary angiography in patients with known or suspected coronary artery disease $e^{2,3}$ or for the follow-up of coronary allograft vasculopathy. ${ }^{4}$ Recent technological progresses have led to the introduction of the silicon photomultiplier (SiPM)-based TOF technology. Briefly, it consists of multiple columns of single-photon avalanche photodiodes, which are operated in Geiger mode.

Reprint requests: John Prior, PhD, MD, Department of Nuclear Medicine and Molecular Imaging, CHUV, Rue du Bugnon 46, 1011

Lausanne, Switzerland; John.Prior@chuv.ch

J Nucl Cardiol 2022;29:213-5.

$1071-3581 / \$ 34.00$

Copyright (C) 2020 American Society of Nuclear Cardiology.
The resulting photon from the annihilation produces a charge avalanche after hitting on the cell, leading to the formation of a discrete electrical impulse. In the socalled digital SiPM, the signal of each individual cell is first digitalized, and the timing and energy information is obtained after summation of the corresponding cells that produces a single count. Other aspects of the detector accounts for its performance, such as the length of the scintillator crystal, the coupling time between the scintillator and the photodetector as well as the novelty in the signal processing. Phillips was the first constructor to introduce SiPM technology 2014, followed by General Electric and more recently by Siemens and others. This technology led to improvement of the timing resolution, with TOF performance actually down to 214 picoseconds, as well as to significant improvement of the spatial resolution. ${ }^{5-7}$ This improvement led to better image quality, increased sensitivity for the detection of small lesions with consequently upstaging of the disease in oncologic PET. ${ }^{8}$ Whether this improvement has a significant impact on the accuracy of the evaluation of myocardial perfusion imaging (MPI) is unknown.

This hypothesis has been questioned in the present study by Koenders et al. ${ }^{9}$ The authors investigated the accuracy of digital SiPM (dSiPM) PET/CT as compared to the high-resolution photomultiplier tube (hrPMT) PET/CT in the evaluation of semi-quantitative as well as quantitative MPI. For this purpose, 30 patients underwent 2 sets of rest and regadenoson-induced stress Rubidium (Rb)-82 MPI, beginning with the hrPMT PET/CT followed by the dSiPM PET/CT within 3 weeks. The images were analyzed by two independent nuclear medicine experts for their quality and the presence of possible defects in the semi-quantitative as well as in the quantitative MBF parameters. The authors described an improvement in the image quality using dSiPM PET/CT as compared to hrPMT PET/CT with 
$-10 \%$ in fair, $-10 \%$ in good and $+20 \%$ in excellent quality ( $p=0.03$ ). There was no significant difference in the defect interpretation and interpreter's confidence between both devices $(p>0.50)$. However, no significant differences in $\operatorname{rMBF}(p \geq 0.29)$, $\operatorname{sMBF}(p \geq 0.11)$, and myocardial flow reserve (MFR) $(p \geq 0.51)$ were observed. The authors concluded that dSiPM PET/CT provided better image quality with comparable defect interpretation and interpreter's confidence, however, without significant difference in absolute MBF and MFR, which made it a reliable technique for the assessment of MPI using Rb-82. We would like to congratulate the authors for this novel, well conducted study providing first evidences about the accuracy of $\mathrm{dSiPM}$ PET/CT in MPI with Rb-82.

In their integration of noninvasive flow parameters with clinical PET-verified ischemic threshold, Johnson and Gould mentioned the necessity of considering the variation of rMBF depending on the pressure rate product (PRP), calculated as the product of systolic blood pressure and heart rate. In a series of about 1500 cardiac PET/CT, they demonstrated that PRP correlates significantly with whole left ventricular MBF, stronger at rest than during maximal coronary vasodilation, accounting for $40 \%$ of the variation of rMBF. ${ }^{10,11}$ Since the authors did not adjust rMBF to PRP in both sets of exams, the variation of MFR, which is the ratio of $\mathrm{SMBF}$ to $\mathrm{rMBF}$, could be the result of this relationship between PRP and myocardial flow in baseline conditions. Since $25 \%$ of patients with intermediate risk based on MFR were reclassified to low/high risk, there are clinical implications of these variations, which should be considered when interpreting absolute MBF parameters. The authors mentioned the large test-retest precision in absolute MBF parameters of $21 \%$ to partly explain this variation. ${ }^{12}$ However, this study was performed on a 2dimensional PET/CT that differs from the 3-dimensional dSiPM PET/CT with higher counting rates, particularly important for MBF quantification when using Rb-82. Despite this limitation, a modest direct linear relation of rMBF to PRP has been found, in accordance to previous published studies. ${ }^{10,11}$ Interestingly, Kitkungvan et al. found that from the $21 \%$ variability observed on repeated rest perfusion measurements on different days, imprecision of the methodology accounts for $10.7 \%$, whereas the rest results from biological variability. Therefore, situations such as anxiety, use of medications such as beta-blockers, use of caffeine or labile systolic blood pressure between the 2 sets of exams could have induce differences in PRP with variation of the resting perfusion, and therefore, from the MFR.

When further questioning the accuracy of dSiPM PET/CT comparing to hrPMT PET/CT, the specific results obtained in two patients challenge us at different levels. These were the patients with discordant results in both series of PET/CT, with presence of ischemia in dSiPM PET/CT that was not seen in hrPMT PET/CT. One of these patients did not undergo further coronary investigation. However, there were no cardiovascular events after a one-year follow-up. For the other one, lesions in coronary angiography, which correlate with the finding of the dSiPM PET/CT have been reported. The authors emphasize about different pitfalls in quantitative myocardial PET perfusion, which should be systematically checked while interpreting MPI images: the correct temporal sampling of the radiotracer infusion with characteristic curves, the correct co-registration of PET and CT for scatter correction, the correct image reconstruction and post-filtering based on evidences and the standardize tracer kinetic modelling. ${ }^{13}$ However, even if all these pitfalls were excluded, we could not conclude on the superiority of $\mathrm{dSiPM}$ PET/CT over hrPMT PET/CT due to the small sample size, which is in line with the author's analysis. To better clarify this hypothesis, future studies should be designed with more patients. Nevertheless, it is quite reassuring to have a concordance in the results of both PET/CT in more than $90 \%$ of the subjects, suggesting that we are still good enough in the evaluation of MPI using Rb-82 with PMT PET/CT as compared to SiPM PET/CT. Since each SiPM PET design has his own particularities, which can be determinant in the acquisition and processing of MPI, it would be difficult to generalize this observation on others SiPM systems. ${ }^{5-7}$ But independently of the SiPM system, one of the advantages we could expect from this technology is the significant radiation dose reduction without alteration of the visual quality as well as the absolute myocardial flow quantification. ${ }^{14}$

\section{Disclosures}

Christel H. Kamani and John O. Prior declare that they have no conflict of interest.

\section{References}

1. Jones T, Townsend D. History and future technical innovation in positron emission tomography. J Med Imaging (Bellingham) 2017;4:011013

2. Knuuti J, Ballo H, Juarez-Orozco LE, Saraste A, Kolh P, Rutjes AWS et al. The performance of non-invasive tests to rule-in and rule-out significant coronary artery stenosis in patients with stable angina: a meta-analysis focused on post-test disease probability. Eur Heart J 2018;39:3322-30

3. Bober RM, Milani RV, Oktay AA, Javed F, Polin NM, Morin DP. The impact of revascularization on myocardial blood flow as assessed by positron emission tomography. Eur J Nucl Med Mol Imaging 2019;46:1226-39

4. Miller RJH, Manabe O, Tamarappoo B, Hayes S, Friedman JD, Slomka PJ et al. Comparative prognostic and diagnostic value of 
myocardial blood flow and myocardial flow reserve after cardiac transplantation. J Nucl Med 2020;61:249-55

5. van Sluis J, de Jong J, Schaar J, Noordzij W, van Snick P, Dierckx $\mathrm{R}$ et al. Performance characteristics of the digital biograph vision PET/CT system. J Nucl Med 2019;60:1031-6

6. Rausch I, Ruiz A, Valverde-Pascual I, Cal-Gonzalez J, Beyer T, Carrio I. Performance evaluation of the vereos PET/CT system according to the NEMA NU2-2012 standard. J Nucl Med 2019;60:561-7

7. Hsu DFC, Ilan E, Peterson WT, Uribe J, Lubberink M, Levin CS. Studies of a next-generation silicon-photomultiplier-based timeof-flight PET/CT system. J Nucl Med 2017;58:1511-8

8. Koopman D, van Dalen JA, Stevens H, Slump CH, Knollema S, Jager PL. Performance of digital PET compared to high-resolution conventional PET in patients with cancer. J Nucl Med 2020. h ttps://doi.org/10.2967/jnumed.119.238105

9. Koenders SS, van Dalen JA, Jager PL, Knollema S, Timmer JR, Mouden $\mathrm{M}$ et al. Value of in myocardial perfusion imaging using Rubidium-82. J Nucl Cardiol 2020.

10. Johnson NP, Gould KL. Physiological basis for angina and STsegment change PET-verified thresholds of quantitative stress myocardial perfusion and coronary flow reserve. JACC Cardiovasc Imaging 2011;4:990-8
11. Johnson NP, Gould KL. Integrating noninvasive absolute flow, coronary flow reserve, and ischemic thresholds into a comprehensive map of physiological severity. JACC Cardiovasc Imaging 2012;5:430-40

12. Kitkungvan D, Johnson NP, Roby AE, Patel MB, Kirkeeide R, Gould KL. Routine clinical quantitative rest stress myocardial perfusion for managing coronary artery disease: Clinical relevance of test-retest variability. JACC Cardiovasc Imaging 2017;10:56577

13. Moody JB, Lee BC, Corbett JR, Ficaro EP, Murthy VL. Precision and accuracy of clinical quantification of myocardial blood flow by dynamic PET: A technical perspective. J Nucl Cardiol 2015;22:935-51

14. DeKemp R, Genoud P, Pappon M, Kamani C, Gnesin S, Prior JO. Dynamic range of the newest digital PET-CT scanner for myocardial blood flow quantification using Rubidium-82. J Nucl Med 2020 (Accepted Abstract, SNMMI New Orleans).

Publisher's Note Springer Nature remains neutral with regard to jurisdictional claims in published maps and institutional affiliations. 